\title{
Challenges in truncating the hierarchy of time-dependent reduced density matrices equations
}

\author{
A. Akbari, ${ }^{1, *}, \dagger$ M. J. Hashemi, ${ }^{2, \dagger}$ A. Rubio, ${ }^{1}$ R. M. Nieminen, ${ }^{2}$ and R. van Leeuwen ${ }^{3}$ \\ ${ }^{1}$ Nano-Bio Spectroscopy Group and ETSF Scientific Development Centre, Departamento de Física de Materiales, Centro de Física de \\ Materiales CSIC-UPVIEHU-MPC and DIPC, Universidad del País Vasco UPVIEHU, Avenida Tolosa 72, E-20018 San Sebastián, Spain \\ ${ }^{2}$ Department of Applied Physics, Aalto University, P.O. Box 11100, FI-00076 Aalto, Finland \\ ${ }^{3}$ Department of Physics, Nanoscience Center, University of Jyväskylä, FI-40014 Jyväskylä, Finland
}

(Received 21 April 2012; published 12 June 2012)

\begin{abstract}
In this work, we analyze the Born, Bogoliubov, Green, Kirkwood, and Yvon (BBGKY) hierarchy of equations for describing the full time evolution of a many-body fermionic system in terms of its reduced density matrices (at all orders). We provide an exhaustive study of the challenges and open problems linked to the truncation of such a hierarchy of equations to make them practically applicable. We restrict our analysis to the coupled evolution of the one- and two-body reduced density matrices, where higher-order correlation effects are embodied into the approximation used to close the equations. We prove that within this approach, the number of electrons and total energy are conserved, regardless of the employed approximation. Further, we demonstrate that although most of the truncation schemes available in the literature give acceptable ground-state energy, when applied to describe driven electron dynamics, they exhibit undesirable and unphysical behavior, e.g., violation and even divergence in local electronic density, both in weakly and strongly correlated regimes. We illustrate and analyze these problems within the few-site Hubbard model. The model can be solved exactly and provides a unique reference for our detailed study of electron dynamics for different values of interaction, different initial conditions, and the large set of approximations considered here. Moreover, we study the role of compatibility between two hierarchical equations and positive semidefiniteness of reduced density matrices in the instability of electron dynamics. We show that even if the used approximation holds the compatibility, electron dynamics can still diverge when positive definitiveness is violated. We propose some partial solutions of such problems and point out the main paths for future work in order to make this approach applicable for the description of the correlated electron dynamics in complex systems.
\end{abstract}

DOI: 10.1103/PhysRevB.85.235121

PACS number(s): 71.10.-w, 71.27.+a

\section{INTRODUCTION}

The description of dynamical processes in many-electron systems brought out of equilibrium requires a proper description of static and dynamical correlation effects. Theoretical study of many fundamental processes of interest, such as attosecond dynamics, high-intensity laser phenomena, lightinduced phase transitions, harmonic generations, etc., requires a proper theoretical framework which is both accurate and tractable.

Time-dependent Hartree-Fock (TDHF) theory ${ }^{1-3}$ was among the first methods devised to simulate dynamics of many-body systems. More advanced methods, such as Kadanoff-Baym equations ${ }^{4,5}$ and the Keldysh technique ${ }^{6}$ for the Green's function, enable us to study nonequilibrium phenomena more accurately, once an approximation for the electron self-energy is chosen properly. They are, however, computationally very demanding. ${ }^{7,8}$

Alternatively, time-dependent density functional theory $(\text { TDDFT })^{9,10}$ is becoming a popular option for its performance, accuracy, and potential scalability. It describes electron dynamics in terms of the electronic density by mapping an interacting system to an auxiliary noninteracting system (Kohn-Sham system) with an effective potential that produces the same time-dependent density. This is ideal for massively parallel implementations as in its time-dependent Kohn-Sham (KS) formulation the evolution of each of the KS orbitals is nearly independent of the others. However, the lack of proper exchange-correlation (xc) functionals for time-dependent systems hampers its applicability. Most of the time-dependent approximations that are used so far are adiabatic extensions of DFT approximations that disregard the nonlocality and memory dependence of the time-dependent xc potential.

In this situation, reduced density matrix (RDM) theories offer a promising framework to deal with time-dependent phenomena. The equation of motion for each RDM can be straightforwardly derived from the time-dependent Schrödinger equation which contains the corresponding and one-order-higher RDM. The whole set of these interrelated equations form the so-called BBGKY hierarchy since a basically similar hierarchy was initially invented and developed by Born, Bogoliubov, Green, Kirkwood, and Yvon ${ }^{11-14}$ in classical statistical mechanics.

As it is not possible to confront the whole hierarchy, one must truncate it at some level. For instance, if one approximates a two-body RDM in terms of a one-body RDM in the first equation, one arrives at the time-dependent version of reduced density matrix functional theory (TD-RDMFT). Similar to the TDDFT, most of the approximations used in the TD-RDMFT are adiabatic extensions of the existing ground-state ones, ${ }^{15-17}$ and even though they can successfully describe the ground state of some strongly correlated systems, ${ }^{18,19}$ they suffer from flaws, such as lack of memory when we apply them in the time domain. As an example, assuming that these approximations have even the right form, the sign adopted in each term generally becomes a time-dependent phase when we extend them over the time domain. Ignoring this fact by using fixed signs may cause problems, such as time-independent occupation numbers. ${ }^{20}$ Furthermore, the majority of these 
approximations do not necessarily conserve the total energy of a system.

Some of these deficiencies will be solved if we consider propagating the first two equations of the hierarchy by approximating the three-body RDM. However, this has been proven to be a nontrivial task, and in fact earlier attempts in nuclear dynamics $^{21,22}$ show that these coupled equations can violate the inequalities related to probabilistic interpretation of RDMs for fermions. For example, the eigenvalues of a one-body RDM must remain, in principle, between 0 and $1,{ }^{23}$ but in practice they violate these bounds, showing the nonfermionic nature of the corresponding RDM. Such behaviors were unexpected, and they were claimed to be related to the violation of the relations between different orders of reduced density matrices.

In this paper, we study in detail the performance of such an approach for different truncation schemes and show that the truncated set of equations may lead to instability and, in many cases, even divergence (in electronic density, occupation numbers, etc.). We mention the specific properties of approximations that are responsible for these unphysical results. We will show that lack of properties such as positive semidefiniteness also plays a crucial role in this failure. In addition, this study prompts one to be aware of the same issues which may arise in building approximations in TD-RDMFT. This paper is divided into three sections. In the next section, we give the theoretical background for the BBGKY hierarchy and different approximations to truncate it. In Sec. III we present the results and analyze the divergence phenomenon by using different approximations, and finally, we conclude the work in Sec. IV.

\section{THEORETICAL BACKGROUND}

\section{A. $N$-particle system and reduced density matrix: Definitions and properties}

First, in order to have a compact notation, we introduce two collections of space-spin coordinates as

$$
X_{n} \equiv\left(\mathbf{x}_{1} \ldots \mathbf{x}_{n}\right) ; \quad \breve{X}_{n} \equiv\left(\mathbf{x}_{n+1} \ldots \mathbf{x}_{N}\right) .
$$

In this notation, $\Phi\left(X_{N}, t\right)$ denotes the normalized wave function of the system. Here, we note that throughout this work we employ atomic units (a.u.).

Now, we consider a system with $N$ identical particles described by the time-dependent Hamiltonian

$$
\hat{H}(t)=\sum_{i=1}^{N} \hat{h}_{i}+\frac{1}{2} \sum_{i \neq j}^{N} U_{i j}
$$

The last term describes the two-particle interactions $U_{i j} \equiv$ $U\left(\mathbf{x}_{i} \mathbf{x}_{j}\right)$, where $\mathbf{x}$ includes both the space coordinates $\mathbf{r}$ and the spin coordinates $\sigma$ of the particles. Usually, $U$ is spin independent and has the form $U\left(\mathbf{x} \mathbf{x}^{\prime}\right)=w\left(\left|\mathbf{r}-\mathbf{r}^{\prime}\right|\right)$. The onebody part, $\hat{h}_{i} \equiv \hat{h}\left(\mathbf{x}_{i}, t\right)$, will be time-dependent and of the form

$$
h(\mathbf{x}, t)=-\frac{1}{2} \nabla^{2}+v(\mathbf{x}, t),
$$

where $v$ is a general time-dependent external field. We can define the $n$-body reduced density matrix $\Gamma^{(n)}$ of such system as

$$
\Gamma^{(n)}\left(X_{n}, X_{n}^{\prime}, t\right)=\frac{N !}{(N-n) !} \int d \breve{X}_{n} \Phi\left(X_{n}, \breve{X}_{n}, t\right) \Phi^{*}\left(X_{n}^{\prime}, \breve{X}_{n}, t\right),
$$

where $d \breve{X}_{n} \equiv d \mathbf{x}_{n+1} \cdots d \mathbf{x}_{N}$ and $\int d \mathbf{x}=\sum_{\sigma} \int d \mathbf{r}$.

Based on the above definition, several important properties of RDMs follow. First, different levels of RDMs are connected to each other by

$$
\int d \mathbf{x}_{n+1} \Gamma^{(n+1)}\left(X_{n} \mathbf{x}_{n+1}, X_{n}^{\prime} \mathbf{x}_{n+1}, t\right)=(N-n) \Gamma^{(n)}\left(X_{n}, X_{n}^{\prime}, t\right),
$$

which we refer to as the partial trace relation. Consequently, if $\Gamma^{(n)}$ is available, all RDMs with lower order can be calculated straightforwardly.

Another important feature is that Eq. (4) implies all RDMs are positive semidefinite, which refers to the fact that all eigenvalues of RDMs are always equal to or greater than zero. For a given order RDM, these eigenvalues and their corresponding eigenvectors can be calculated as

$$
\int d X_{n}^{\prime} \Gamma^{(n)}\left(X_{n}, X_{n}^{\prime}, t\right) g_{i}\left(X_{n}^{\prime}, t\right)=\lambda_{i}(t) g_{i}\left(X_{n}, t\right), \quad \lambda_{i}(t) \geqslant 0 .
$$

Conventionally, the eigenvectors and eigenvalues of $\Gamma^{(1)}$ are called natural orbitals and natural orbital occupation numbers, respectively, and those of $\Gamma^{(2)}$ are called geminals and geminal occupation numbers, respectively.

Moreover, in the case of fermionic particles, the Pauli exclusion principle enforces natural orbital occupation numbers to be less than or equal to $1^{23}$ Thus, they have to remain between 0 and 1 . This corresponds to the fermionic inequality.

\section{B. Equation of motion, BBGKY hierarchy, and conservation laws}

Provided the initial state of the system is given, its time evolution is completely described by the time-dependent Schrödinger equation (TDSE). Nonetheless, the solution of the TDSE is not usually possible, except for systems with few particles, due to the many degrees of freedom of the system. In contrast, most quantities of interest are $n$-body observables which can be obtained from the $n$-body reduced density matrix $\Gamma^{(n)}$, and in most systems of relevance it holds that $n \ll N$. It is therefore natural to derive the equations of motion for reduced density matrices. Thus, using Eq. (4) together with the TDSE, we get

$$
\begin{aligned}
\left(i \partial_{t}\right. & \left.-\hat{H}_{1 \cdots n}+\hat{H}_{1^{\prime} \cdots n^{\prime}}\right) \Gamma^{(n)}\left(X_{n}, X_{n}^{\prime}, t\right) \\
= & \sum_{i=1}^{n} \int d \mathbf{x}_{n+1}\left[U\left(\mathbf{x}_{i} \mathbf{x}_{n+1}\right)-U\left(\mathbf{x}_{i}^{\prime} \mathbf{x}_{n+1}\right)\right] \\
& \times \Gamma^{(n+1)}\left(X_{n} \mathbf{x}_{n+1}, X_{n}^{\prime} \mathbf{x}_{n+1}, t\right),
\end{aligned}
$$

where we defined

$$
\hat{H}_{1 \cdots n}=\sum_{i=1}^{n} \hat{h}_{i}+\frac{1}{2} \sum_{i \neq j}^{n} U_{i j} .
$$


The entire set of $N$ equations for reduced density matrices forms the well-known BBGKY hierarchy. The explicit forms of the first two equations are

$$
\begin{aligned}
& \left(i \partial_{t}-\hat{h}_{1}+\hat{h}_{1^{\prime}}\right) \gamma\left(\mathbf{x}_{1}, \mathbf{x}_{1}^{\prime}, t\right) \\
& \quad=\int d \mathbf{x}_{2}\left[U\left(\mathbf{x}_{1} \mathbf{x}_{2}\right)-U\left(\mathbf{x}_{1}^{\prime} \mathbf{x}_{2}\right)\right] \Gamma\left(\mathbf{x}_{1} \mathbf{x}_{2}, \mathbf{x}_{1}^{\prime} \mathbf{x}_{2}, t\right)
\end{aligned}
$$

and

$$
\begin{aligned}
& \left(i \partial_{t}-\hat{H}_{12}+\hat{H}_{1^{\prime} 2^{\prime}}\right) \Gamma\left(\mathbf{x}_{1} \mathbf{x}_{2}, \mathbf{x}_{1}^{\prime} \mathbf{x}_{2}^{\prime}, t\right) \\
& =\int d \mathbf{x}_{3}\left[U\left(\mathbf{x}_{1} \mathbf{x}_{3}\right)+U\left(\mathbf{x}_{2} \mathbf{x}_{3}\right)-U\left(\mathbf{x}_{1}^{\prime} \mathbf{x}_{3}\right)-U\left(\mathbf{x}_{2}^{\prime} \mathbf{x}_{3}\right)\right] \\
& \quad \times \Gamma^{(3)}\left(\mathbf{x}_{1} \mathbf{x}_{2} \mathbf{x}_{3}, \mathbf{x}_{1}^{\prime} \mathbf{x}_{2}^{\prime} \mathbf{x}_{3}, t\right)
\end{aligned}
$$

where $\gamma \equiv \Gamma^{(1)}$ and $\Gamma \equiv \Gamma^{(2)}$. As is customary in the literature, ${ }^{24}$ we call the right-hand side of Eq. (10) the three-body collision integral and use $S$ to refer to it. In general, each equation of the hierarchy relates a given-order of RDM, $\Gamma^{(n)}$, to the one-order-higher RDM, $\Gamma^{(n+1)}$. In order to make the BBGKY hierarchy practical, we must truncate it at some level, $n$, by reconstructing $\Gamma^{(n+1)}$ as a functional of lower-order RDMs. Although such a reconstruction in terms of the one-body time-dependent density matrix is, in principle, conceivable by virtue of the Runge-Gross theorem, ${ }^{9,25}$ there is no practical method available to find the exact functional, and we have to use different approximations.

In this work, however, we will only propagate $\gamma$ and $\Gamma$ since they are sufficient to calculate the dynamics of all oneand two-body observables. For instance, for the case of total energy, the expectation values of the one- and two-body parts of the Hamiltonian, $E_{1}(t)$ and $E_{2}(t)$, can be written as

$$
E_{1}(t)=\sum_{i}^{N}\left\langle\hat{h}_{i}\right\rangle=\left.\int d \mathbf{x}^{\prime} h\left(\mathbf{x}^{\prime}, t\right) \gamma\left(\mathbf{x}, \mathbf{x}^{\prime}, t\right)\right|_{\mathbf{x}=\mathbf{x}^{\prime}}
$$

and

$$
E_{2}(t)=\frac{1}{2} \sum_{i, j}^{N}\left\langle U_{i j}\right\rangle=\frac{1}{2} \int d X_{2} U\left(X_{2}\right) \Gamma\left(X_{2}, X_{2}, t\right) .
$$

To this end, we need to truncate Eq. (10) by approximating $\Gamma^{(3)}$ in terms of $\gamma$ and $\Gamma$. Several of these approximations will be discussed shortly.

At this point we highlight an important property of the BBGKY hierarchy and the effect of truncation on it. As a direct outcome of Eq. (5), different levels of the hierarchy are compatible; namely, equations in the higher levels of the hierarchy are reducible to the lower-level ones. We refer to this link between equations as the compatibility condition that preferably should be fulfilled by a good approximation. Thus, compatibility signifies that the highest equation is equivalent to the whole BBGKY hierarchy. This is not surprising since the highest equation is basically the original Schrödinger equation. However, when we truncate the hierarchy by introducing an approximation for $\Gamma^{(3)}$, the partial trace relation between $\Gamma^{(3)}$ and $\Gamma$ does not necessarily hold, and thus it generally breaks the compatibility between Eqs. (9) and (10) (see Sec. III C for some exceptions). Consequently, when we truncate the BBGKY hierarchy, we have two generally distinct options to propagate the equations which should be equivalent if the truncation approximation satisfies compatibility. (i) Propagating two coupled equations. We can evolve both $\gamma$ and $\Gamma$ by solving Eqs. (9) and (10) together as coupled equations since the two equations most likely are no longer compatible after approximating $\Gamma^{(3)}$ in Eq. (10).

(ii) Propagating only the second equation. To avoid the problem of compatibility between two equations, we can evolve only Eq. (10). Then we assign $\gamma$ to be the partial trace of $\Gamma$ and denote it as $\gamma_{\Gamma}$ to distinguish it from general $\gamma$. It mathematically reads

$$
\gamma_{\Gamma}\left(\mathbf{x}_{1}, \mathbf{x}_{1}^{\prime}, t\right)=\frac{1}{N-1} \int d \mathbf{x}_{2} \Gamma\left(\mathbf{x}_{1} \mathbf{x}_{2}, \mathbf{x}_{1}^{\prime} \mathbf{x}_{2}, t\right) .
$$

In this way, we prevent the complication of dealing with two coupled equations.

The difference between these two approaches lies in the distinction between $\gamma$ and $\gamma_{\Gamma}$. To see this, we derive the equation of motion for Eq. (13) by using Eq. (10). We have

$$
\begin{aligned}
i \partial_{t} \gamma_{\Gamma}\left(\mathbf{x}_{1}, \mathbf{x}_{1}^{\prime}, t\right)= & \left.\frac{1}{N-1} \int d \mathbf{x}_{2} i \partial_{t} \Gamma\left(X_{2}, X_{2}^{\prime}, t\right)\right|_{\mathbf{x}_{2}=\mathbf{x}_{2}^{\prime}} \\
= & \left.\frac{1}{N-1} \int d \mathbf{x}_{2}\left(H_{12}-H_{1^{\prime} 2^{\prime}}\right) \Gamma\left(X_{2}, X_{2}^{\prime}, t\right)\right|_{\mathbf{x}_{2}=\mathbf{x}_{2}^{\prime}} \\
& +\frac{1}{N-1} \int d \mathbf{x}_{2} d \mathbf{x}_{3}\left[U\left(\mathbf{x}_{1} \mathbf{x}_{3}\right)-U\left(\mathbf{x}_{1} \mathbf{x}_{3}\right)\right] \\
& \times \Gamma_{\text {app }}^{(3)}\left(\mathbf{x}_{1} \mathbf{x}_{2} \mathbf{x}_{3}, \mathbf{x}_{1}^{\prime} \mathbf{x}_{2} \mathbf{x}_{3}, t\right),
\end{aligned}
$$

where the approximated three-body $\operatorname{RDM} \Gamma_{\text {app }}^{(3)}$ does not necessarily integrate to $(N-2) \Gamma$ as we pointed out earlier. Now, using the explicit form of $H_{12}$, we can rewrite Eq. (14) as

$$
\begin{aligned}
i \partial_{t} \gamma_{\Gamma} & \left(\mathbf{x}_{1}, \mathbf{x}_{1}^{\prime}, t\right) \\
= & \left(h_{1}-h_{1^{\prime}}\right) \gamma_{\Gamma}\left(\mathbf{x}_{1}, \mathbf{x}_{1}^{\prime}, t\right) \\
& +\int d \mathbf{x}_{2}\left[U\left(\mathbf{x}_{1} \mathbf{x}_{2}\right)-U\left(\mathbf{x}_{1}^{\prime} \mathbf{x}_{2}\right)\right] \bar{\Gamma}\left(X_{2}, X_{2}, t\right) \\
& -\left.\frac{1}{2} \int d \mathbf{x}_{2}\left(\nabla_{2}^{2}-\nabla_{2}^{\prime 2}\right) \Gamma\left(X_{2}, X_{2}^{\prime}, t\right)\right|_{\mathbf{x}_{2}=\mathbf{x}_{2}^{\prime},}
\end{aligned}
$$

where

$$
\begin{aligned}
\bar{\Gamma}\left(X_{2}, X_{2}^{\prime}, t\right)= & \frac{1}{N-1}\left[\Gamma\left(X_{2}, X_{2}^{\prime}, t\right)\right. \\
& \left.+\int d \mathbf{x}_{3} \Gamma_{\text {app }}^{(3)}\left(\mathbf{x}_{1} \mathbf{x}_{2} \mathbf{x}_{3}, \mathbf{x}_{1}^{\prime} \mathbf{x}_{2}^{\prime} \mathbf{x}_{3}, t\right)\right] .
\end{aligned}
$$

The last term in Eq. (15) can be written as a total divergence as follows:

$$
\begin{aligned}
& \left.\left(\nabla_{2}^{2}-\nabla_{2}^{\prime 2}\right) \Gamma\left(X_{2}, X_{2}^{\prime}, t\right)\right|_{\mathbf{x}_{2}=\mathbf{x}_{2}^{\prime}} \\
& \quad=\nabla_{2} \cdot\left[\left.\left(\nabla_{2}-\nabla_{2}^{\prime}\right) \Gamma\left(X_{2}, X_{2}^{\prime}, t\right)\right|_{\mathbf{x}_{2}=\mathbf{x}_{2}^{\prime}}\right],
\end{aligned}
$$

and hence it vanishes after integration over $\mathbf{x}_{2}$. Finally, Eq. (15) becomes

$$
\begin{aligned}
& \left(i \partial_{t}-\hat{h}_{1}+\hat{h}_{1^{\prime}}\right) \gamma_{\Gamma}\left(\mathbf{x}_{1}, \mathbf{x}_{1}^{\prime}, t\right) \\
& \quad=\int d \mathbf{x}_{2}\left[U\left(\mathbf{x}_{1} \mathbf{x}_{2}\right)-U\left(\mathbf{x}_{1}^{\prime} \mathbf{x}_{2}\right)\right] \bar{\Gamma}\left(\mathbf{x}_{1} \mathbf{x}_{2}, \mathbf{x}_{1}^{\prime} \mathbf{x}_{2}, t\right) .
\end{aligned}
$$

Obviously, the equations of motion for $\gamma_{\Gamma}$ and $\gamma$, Eqs. (18) and (9), respectively, will be equivalent if $\bar{\Gamma}=\Gamma$. This derivation shows that Eqs. (9) and (18), and therefore the two above-mentioned approaches, are generally different because $\Gamma_{\mathrm{app}}^{(3)}$ does not necessarily integrate to $(N-2) \Gamma$. 
It is a priori not clear which of the two approaches is preferable. For that, let us examine if the approximate equations maintain important properties such as particle number and energy conservation.

\section{Particle number conservation}

First, we discuss the particle number conservation. To do this, we note that the diagonal of the one-body RDM, $\gamma(\mathbf{x}, \mathbf{x}, t)$, gives the particle density $n(\mathbf{x}, t)$. Now, particle number conservation means that the total number of particles, $N(t)=\int d \mathbf{x} n(\mathbf{x}, t)$, is independent of time, i.e., $\partial_{t} N(t)=0$. This is guaranteed once the continuity equation holds. In fact, this is the case for both approaches since from either Eq. (9) or Eq. (18) we arrive at

$$
\begin{aligned}
\partial_{t} n(\mathbf{x}, t) & =\left.\partial_{t} \gamma\left(\mathbf{x}, \mathbf{x}^{\prime}, t\right)\right|_{\mathbf{x}^{\prime}=\mathbf{x}} \\
& =-\left.\frac{1}{2 i}\left(\nabla^{2}-\nabla^{\prime 2}\right) \gamma\left(\mathbf{x}, \mathbf{x}^{\prime}, t\right)\right|_{\mathbf{x}^{\prime}=\mathbf{x}} \\
& =-\left.\frac{1}{2 i}\left(\nabla^{2}+\nabla \cdot \nabla^{\prime}-\nabla \cdot \nabla^{\prime}-\nabla^{\prime 2}\right) \gamma\left(\mathbf{x}, \mathbf{x}^{\prime}, t\right)\right|_{\mathbf{x}^{\prime}=\mathbf{x}} \\
& =-\nabla \cdot\left[\left.\frac{1}{2 i}\left(\nabla-\nabla^{\prime}\right) \gamma\left(\mathbf{x}, \mathbf{x}^{\prime}, t\right)\right|_{\mathbf{x}^{\prime}=\mathbf{x}}\right] \\
& =-\nabla \cdot \mathbf{j}(\mathbf{x}, t)
\end{aligned}
$$

where we defined the current density $\mathbf{j}(\mathbf{x}, t)$ as the quantity inside the braces. This follows immediately from the fact that the right-hand side of both Eqs. (9) and (18) vanishes for $\mathbf{x}_{1}=$ $\mathbf{x}_{1}^{\prime}$ independent of $\Gamma$ or $\bar{\Gamma}$. Thus, the total number of particles is preserved even if we cut the hierarchy at the first level.

\section{Energy conservation}

Now we turn to the energy conservation. The total energy of the system is the sum of Eqs. (11) and (12). For the second approach where we use only Eq. (10), the time derivative of the total energy after some algebra (see Appendix A) becomes

$$
\begin{aligned}
\frac{d E}{d t}= & \int d \mathbf{x} \partial_{t} v(\mathbf{x}, t) n(\mathbf{x}, t)+\frac{1}{2 i} \int d \mathbf{x}_{1} d \mathbf{x}_{2} \nabla_{1} U\left(\mathbf{x}_{1} \mathbf{x}_{2}\right) \\
& \cdot\left\{\left.\left(\nabla_{1}-\nabla_{1^{\prime}}\right)\left[\Gamma\left(\mathbf{x}_{1} \mathbf{x}_{2}, \mathbf{x}_{1}^{\prime} \mathbf{x}_{2}, t\right)-\bar{\Gamma}\left(\mathbf{x}_{1} \mathbf{x}_{2}, \mathbf{x}_{1}^{\prime} \mathbf{x}_{2}, t\right)\right]\right|_{1=1^{\prime}}\right\} .
\end{aligned}
$$

In the absence of time-dependent potentials $v$, this equation gives $d E / d t=0$, provided $\Gamma=\bar{\Gamma}$. Nonetheless, as we discussed, this is not generally valid in the second approach, and it means the total energy is not conserved there. In contrast, when we solve both Eqs. (9) and (10) together, the second term of Eq. (20) vanishes automatically [Eq. (A10) in Appendix A], ${ }^{24,26}$ and the energy remains constant.

The energy conservation gives us a strong motivation for preferring the first approach, in which we propagate both equations simultaneously, over the second, and this is what we do in the remainder of this work. However, we look into the other case briefly in Sec. III D and show that the energy can fluctuate relatively largely in time for all the approximations.

\section{Hierarchy truncation methods: Approximating $\Gamma^{(3)}$}

In this section we discuss in detail different truncation schemes that we have evaluated in this work. One systematic way of building these approximations is called cluster expansion, which is a method of reconstructing higher-order RDMs as antisymmetrized products of lower-order ones plus a residual correlation function. ${ }^{26-30}$ To have compact notation, first we define the wedge product as the antisymmetrized product of $p$ - and $m$-point functions by

$$
\begin{aligned}
a\left(X_{p}, X_{p}^{\prime}\right) \wedge b\left(\breve{X}_{p}, \breve{X}_{p}^{\prime}\right) \\
=\left(\frac{1}{N !}\right)^{2} \sum_{\alpha, \beta} \epsilon(\alpha) \epsilon(\beta) a\left(\mathbf{x}_{\alpha_{1}} \cdots \mathbf{x}_{\alpha_{p}}, \mathbf{x}_{\beta_{1}}^{\prime} \cdots \mathbf{x}_{\beta_{p}}^{\prime}\right) \\
\quad \times b\left(\mathbf{x}_{\alpha_{p+1}} \cdots \mathbf{x}_{\alpha_{N}}, \mathbf{x}_{\beta_{p+1}}^{\prime} \cdots \mathbf{x}_{\beta_{N}}^{\prime}\right) .
\end{aligned}
$$

Here, $N=p+m, \alpha$ represents all permutations of the unprimed coordinates, $\beta$ represents all permutations of the primed ones, and the function $\epsilon(\alpha)$ returns +1 when the permutation $\alpha$ contains an even number of transpositions and -1 for an odd number of transpositions. ${ }^{31}$ For instance, the wedge product of two general one-particle matrices is

$$
\begin{aligned}
a\left(\mathbf{x}_{1}, \mathbf{x}_{1}^{\prime}\right) \wedge b\left(\mathbf{x}_{2}, \mathbf{x}_{2}^{\prime}\right) \\
=\frac{1}{4}\left\{a\left(\mathbf{x}_{1}, \mathbf{x}_{1}^{\prime}\right) b\left(\mathbf{x}_{2}, \mathbf{x}_{2}^{\prime}\right)-a\left(\mathbf{x}_{1}, \mathbf{x}_{2}^{\prime}\right) b\left(\mathbf{x}_{2}, \mathbf{x}_{1}^{\prime}\right)\right. \\
\left.\quad+a\left(\mathbf{x}_{2}, \mathbf{x}_{2}^{\prime}\right) b\left(\mathbf{x}_{1}, \mathbf{x}_{1}^{\prime}\right)-a\left(\mathbf{x}_{2}, \mathbf{x}_{1}^{\prime}\right) b\left(\mathbf{x}_{1}, \mathbf{x}_{2}^{\prime}\right)\right\} .
\end{aligned}
$$

Now, we illustrate the cluster expansion with some examples. The first term of the expansion of $\Gamma^{(n)}$ has the same form as in the noninteracting-particle picture; namely, it is an $n$-dimensional determinant of $\gamma$, with $\gamma\left(\mathbf{x}_{i}, \mathbf{x}_{j}^{\prime}, t\right)$ placed in row $i$ and column $j$. For instance, for $\Gamma^{(2)}$, the first term reads

$$
\left|\begin{array}{ll}
\gamma\left(\mathbf{x}_{1}, \mathbf{x}_{1}^{\prime}, t\right) & \gamma\left(\mathbf{x}_{1}, \mathbf{x}_{2}^{\prime}, t\right) \\
\gamma\left(\mathbf{x}_{2}, \mathbf{x}_{1}^{\prime}, t\right) & \gamma\left(\mathbf{x}_{2}, \mathbf{x}_{2}^{\prime}, t\right)
\end{array}\right| \equiv 2 \gamma \wedge \gamma .
$$

Now, we define a two-body correlation function $\Delta^{(2)}$ as the deviation of $\Gamma$ from the noninteracting form such that

$$
\Gamma\left(X_{2}, X_{2}^{\prime}, t\right)=2 \gamma \wedge \gamma+\Delta^{(2)}\left(X_{2}, X_{2}^{\prime}, t\right) .
$$

If we, for instance, approximate $\Gamma_{\text {app }}=2 \gamma \wedge \gamma$ and replace it in the first equation of the BBGKY hierarchy (9), we recover immediately the well-known TDHF equation.

For $\Gamma^{(3)}$, accordingly, we use a noninteracting particle form and add antisymmetrized products of $\gamma$ with the correlation function $\Delta^{(2)}$, which partly describe the three-body correlation, plus a remainder, $\Delta^{(3)}$, i.e.,

$$
\begin{aligned}
\Gamma^{(3)}\left(X_{3}, X_{3}^{\prime}, t\right)= & \left|\begin{array}{lll}
\gamma\left(\mathbf{x}_{1}, \mathbf{x}_{1}^{\prime}, t\right) & \gamma\left(\mathbf{x}_{1}, \mathbf{x}_{2}^{\prime}, t\right) & \gamma\left(\mathbf{x}_{1}, \mathbf{x}_{3}^{\prime}, t\right) \\
\gamma\left(\mathbf{x}_{2}, \mathbf{x}_{1}^{\prime}, t\right) & \gamma\left(\mathbf{x}_{2}, \mathbf{x}_{2}^{\prime}, t\right) & \gamma\left(\mathbf{x}_{2}, \mathbf{x}_{3}^{\prime}, t\right) \\
\gamma\left(\mathbf{x}_{3}, \mathbf{x}_{1}^{\prime}, t\right) & \gamma\left(\mathbf{x}_{3}, \mathbf{x}_{2}^{\prime}, t\right) & \gamma\left(\mathbf{x}_{3}, \mathbf{x}_{3}^{\prime}, t\right)
\end{array}\right| \\
& +\sum_{i, j=1}^{3}(-1)^{i+j} \gamma\left(\mathbf{x}_{i}, \mathbf{x}_{j}^{\prime}, t\right) \Delta^{(2)}\left(\breve{\mathbf{x}}_{i}, \breve{\mathbf{x}}_{j}^{\prime}, t\right) \\
& +\Delta^{(3)}\left(X_{3}, X_{3}^{\prime}, t\right) .
\end{aligned}
$$

In the second term on the right-hand side, $\breve{\mathbf{x}}_{j}$ denotes the pair of variables in the set $\left(\mathbf{x}_{1} \mathbf{x}_{2} \mathbf{x}_{3}\right)$ complementary to $\mathbf{x}_{j}$ keeping the order of the arguments fixed; the same goes for the primed coordinates. For example, $\breve{\mathbf{x}}_{2}=\left(\mathbf{x}_{1} \mathbf{x}_{3}\right)$. Using the wedge product notation, we can rewrite Eq. (25) as

$$
\begin{aligned}
\Gamma^{(3)} & =6 \gamma \wedge \gamma \wedge \gamma+9 \gamma \wedge \Delta^{(2)}+\Delta^{(3)} \\
& =-12 \gamma \wedge \gamma \wedge \gamma+9 \gamma \wedge \Gamma+\Delta^{(3)},
\end{aligned}
$$


in which we replaced $\Delta^{(2)}=\Gamma-2 \gamma \wedge \gamma$ from Eq. (24). Similarly, we can write the expansion for higher-order RDMs.

The same method has been used in the contracted Schrödinger equation formalism (the hierarchical set of equations for density matrices derived from the timeindependent Schrödinger equation) and is referred to as cumulant expansion. ${ }^{32-35}$ Nakatsuji and Yasuda made the expansion more grounded by deriving it using the relation between RDMs and Green's functions. ${ }^{33}$ Based on these, We are now ready to discuss a number of approximations for $\Gamma^{(3)}$.

(i) Three-body collision-integral-free ( $3 b$-CIF) approximation. The simplest one rises from the assumption of $\Gamma^{(3)}=0$, which removes the whole right-hand-side of Eq. (9).

(ii) Three-body noninteracting approximation (3b-NIA). This is obtained only by considering the noninteracting term of Eq. (26):

$$
\Gamma_{3 \mathrm{~b}-\mathrm{NIA}}^{(3)}=6 \gamma \wedge \gamma \wedge \gamma
$$

This gives $\Gamma^{(3)}$ as a functional of $\gamma$.

(iii) WC approximation. We can, of course, move to the next level and take also the second term of Eq. (26) into account, which leads us to

$$
\Gamma_{\mathrm{WC}}^{(3)}=-12 \gamma \wedge \gamma \wedge \gamma+9 \gamma \wedge \Gamma,
$$

where the index stands for Wang and Cassing, who introduced this approximation in $1985 .^{26}$ This properly reduces to Eq. (27) when we assume $\Gamma=2 \gamma \wedge \gamma$.

Now, if we want to go beyond these approximations, $\Delta^{(3)}$ in Eq. (26) must be approximated. In fact, such approximations have been constructed, ${ }^{33-35}$ for instance, by using the connection between RDMs and the Green's functions, ${ }^{33}$ but we are not going to deal with them in this paper.

\section{DISCUSSION OF THE RESULTS}

In order to test the aforementioned approximations, we need a system for which we have access to its exact or nearly exact solution. The Hubbard model fits very well here since we can solve it exactly for a few sites; also, since the number of single-particle orbitals that build the many-body Hilbert space is limited, we can retain the full single-particle basis set and avoid basis-set truncation errors. It is worth mentioning that although here we illustrate only the performance of all the approximations for the Hubbard model, our preliminary results for small molecules also exhibit very similar issues. ${ }^{36}$

Now, we consider the case of a linear finite Hubbard chain with only nearest-neighbor interactions. The Hubbard Hamiltonian for a finite one-dimensional system in second quantization notation is

$$
\hat{H}=\sum_{\sigma, i} t\left(a_{i+1, \sigma}^{\dagger} a_{i, \sigma}+a_{i, \sigma}^{\dagger} a_{i+1, \sigma}\right)+\sum_{i} U n_{i \uparrow} n_{i \downarrow},
$$

where $\sigma$ is a spin index, $i$ is the site index, and $t$ and $U$ denote hopping and on-site Coulomb energy, respectively. Here, $t$ is set to unity, and $U$ gets different values to simulate different correlation strengths.

To study the quality of the approximations, we must go beyond two-particle systems since they can be treated exactly in our formalism. In this work we will avoid the practical complications introduced by spin in odd-number-electron systems and perform all our calculations for four electrons in a four-site system; however, that does not affect the generality of our results. A more detailed discussion on numerical aspects is given in Appendix B, and the code is also available upon request. ${ }^{37}$

In this part, we investigate three different approximations of $\Gamma^{(3)}$, namely, the three-body collision-integral-free, the three-body noninteracting, and the WC approximations, and compare them with the exact and the TDHF results. With these approximations, we have now a closed set of equations, and as with any differential equation, we need an initial state of the system to propagate them. To study the initial-state dependence of the phenomena, we choose two extreme regimes of initial states to perform our calculations: far from equilibrium and close to equilibrium.

At first, we choose a far-from-equilibrium state as our initial state since it helps us to show the problem more clearly. We build such an initial state by putting four electrons in the two leftmost sites, i.e.,

$$
\left|\Psi_{0}\right\rangle=a_{1, \uparrow}^{\dagger} a_{1, \downarrow}^{\dagger} a_{2, \uparrow}^{\dagger} a_{2, \downarrow}^{\dagger}|0\rangle,
$$

where 1 and 2 refer to two neighboring sites at the beginning of the chain. In Appendix B, we give the matrix form of the initial state and Eqs. (9) and (10) that we actually propagate.

Here, the equations in hand are ordinary differential equations, and we solve them numerically using the Runge-Kutta method. However, to ensure the accuracy and stability of our results, we also used more accurate time-propagation schemes, such as the fourth-order Adams-Bashforth-Moulton method, and we found no notable difference (for a detailed discussion of these methods, see Ref. 38).

The time evolution of electronic density in the leftmost site, $n(1, t)$, is plotted in Fig. 1 for weak on-site Coulomb energy, $U=0.1$, and for TDHF [Fig. 1(a)], 3b-CIF [Fig. 1(b)], 3b-NIA [Fig. 1(c)], and the WC approximations [Fig. 1(d)]. The plots also contain the exact result for comparison. On a short time scale, we can see that all three approximations improve the quality of the results considerably compared to the TDHF. However, compared to each other, the approximations do not exhibit large differences.

Figure 2 shows essentially the same results for a longer propagation time. It also shows how the highest and lowest geminal occupation numbers, $\lambda_{\max }$ and $\lambda_{\min }$, behave over time. For the 3b-CIF approximation in Fig. 2(a) we can see unphysical behavior around $t \approx 240$ a.u., where the density acquires negative values or rises beyond two electrons in a site. The problem is more serious for the two other approximations since, for longer propagation times, the electronic density starts to oscillate with amplitudes much beyond physically allowed boundaries and eventually diverges, as shown in Figs. 2(b) and 2(c). The divergence time depends on the correlation strength, namely, on the value of $U$ in our model, and it decreases almost exponentially with increasing $U$. For example, for the WC approximation, the divergence time changes from $t \approx$ 532 a.u. for $U=0.1$ to $t \approx 3$ a.u. for $U=10$. It is important to note that in the $3 b$-NIA and WC approximations, $\lambda_{\max }$ and $\lambda_{\min }$ start to diverge much earlier, although we cannot immediately see the effect in either natural orbital occupation numbers or on-site electronic densities. 

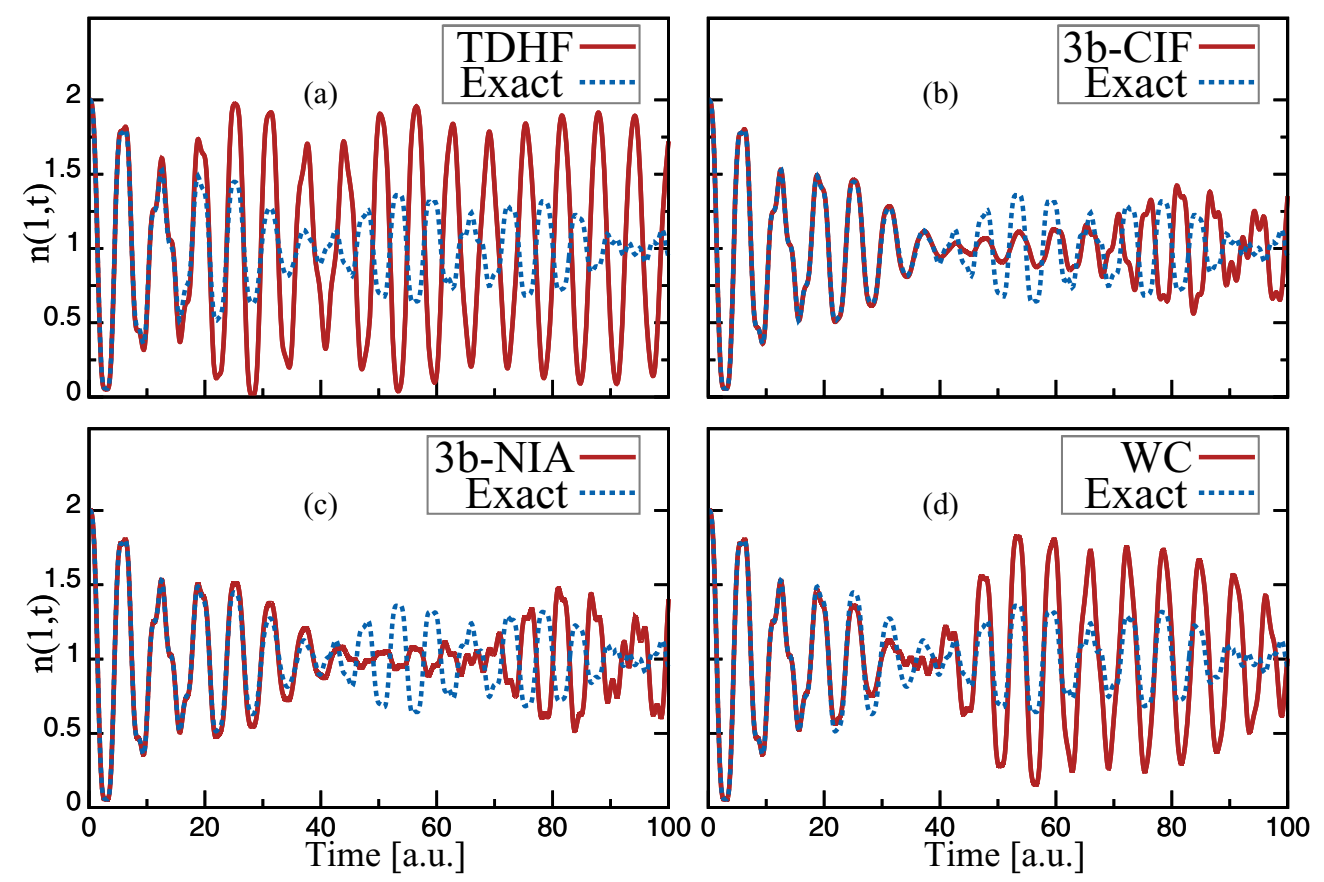

FIG. 1. (Color online) Time evolution of electronic density in the leftmost site of a four-site Hubbard model with the (a) TDHF, (b) 3b-CIF, (c) 3b-NIA, and (d) WC approximations. The exact result is also given for comparison. Here, $m$ and $\hbar$ are set to unity, and Hubbard parameters are $U=0.1$ and $t=1$. The four electrons filled the two leftmost sites initially.

Nonetheless, it is well known that the time evolution of a far-from-equilibrium state is generally very difficult to handle with any approximation, particularly with the ground-statetuned ones; hence, we change the initial states to be closer to the system's ground state in order to investigate the generality of this phenomenon. First, we start the simulation with the initial $\gamma$ and $\Gamma$ extracted from the ground state of (i) the exact solution and (ii) the Hartree-Fock approximation; then we let it propagate with all three different approximations. Although in these cases the electronic density for the 3b-CIF approximation does not violate physical bounds, we still see the divergence for the other two approximations.

Moreover, we used the method introduced by Mazziotti ${ }^{39}$ to find the ground state associated with the 3b-NIA and WC approximations and then used it as the initial state. However, since the method ${ }^{39}$ is not totally convergent, the result is not a truly stationary state, and even starting from such state does not bring stability to the equations, and divergence appears again. We will discuss this issue in more detail in a future presentation. ${ }^{36}$

These tests show that the divergence problem is independent of the initial state and has to do with the nature of the approximated equations. It is worth emphasizing again that in all of these approximations the continuity condition, Eq. (19), has not been violated and the total number of particles is always conserved. Nevertheless, the continuity equation does not guarantee that the electronic density in each state does not go below 0 or beyond 2 .

As we mentioned in the Introduction, the violation of fermionic inequality has also been observed for a different system in nuclear physics. ${ }^{21,22}$ In fact, there are earlier works in the classical BBGKY theory that studied the effect of nonlinearity introduced by truncation of the hierarchy and showed the existence of instability in these coupled equations depending on the initial conditions of the system. ${ }^{40,41}$ Other studies also indicated that the classical collision integral can diverge. ${ }^{42,43}$ Such catastrophic behaviors of these coupled equations pose a valid question of why even highly advanced approximations based on the Green's-function expansion not only fail to follow fundamental physical principles but also lead to divergence, even though the total energy and number of particles are conserved.

To analyze this phenomenon we focus our attention on the basic properties of the BBGKY hierarchy and density matrices to find out how they are affected by different approximations. As we already showed, the employed approximations break the compatibility between Eq. (9) and the approximated version of Eq. (10), and the partial trace relation Eq. (5) between $\Gamma$ and $\gamma$ no longer holds. Schmitt et al. ${ }^{21}$ and Gherega et al. ${ }^{22}$ claimed this was the main reason for the violation of fermionic inequality.

On the other hand, it is obvious that the positive semidefiniteness of density matrices has also been violated. This problem may arise for one of the following reasons.

(i) The approximation functional of $\Gamma^{(3)}$ in Eq. (10) is built in a way that $\Gamma$ does not necessarily stay positive semidefinite, even though the initial $\gamma$ and $\Gamma$ are positive semidefinite (see cases $\mathrm{C}$ and $\mathrm{D}$ below). Then regardless of whether the partial trace relation between $\gamma$ and $\Gamma$ holds or not, there is no guarantee for $\gamma$ to be positive semidefinite.

(ii) The approximation functional of $\Gamma^{(3)}$ is built in a way that the propagated $\Gamma$ does stay positive semidefinite (provided 

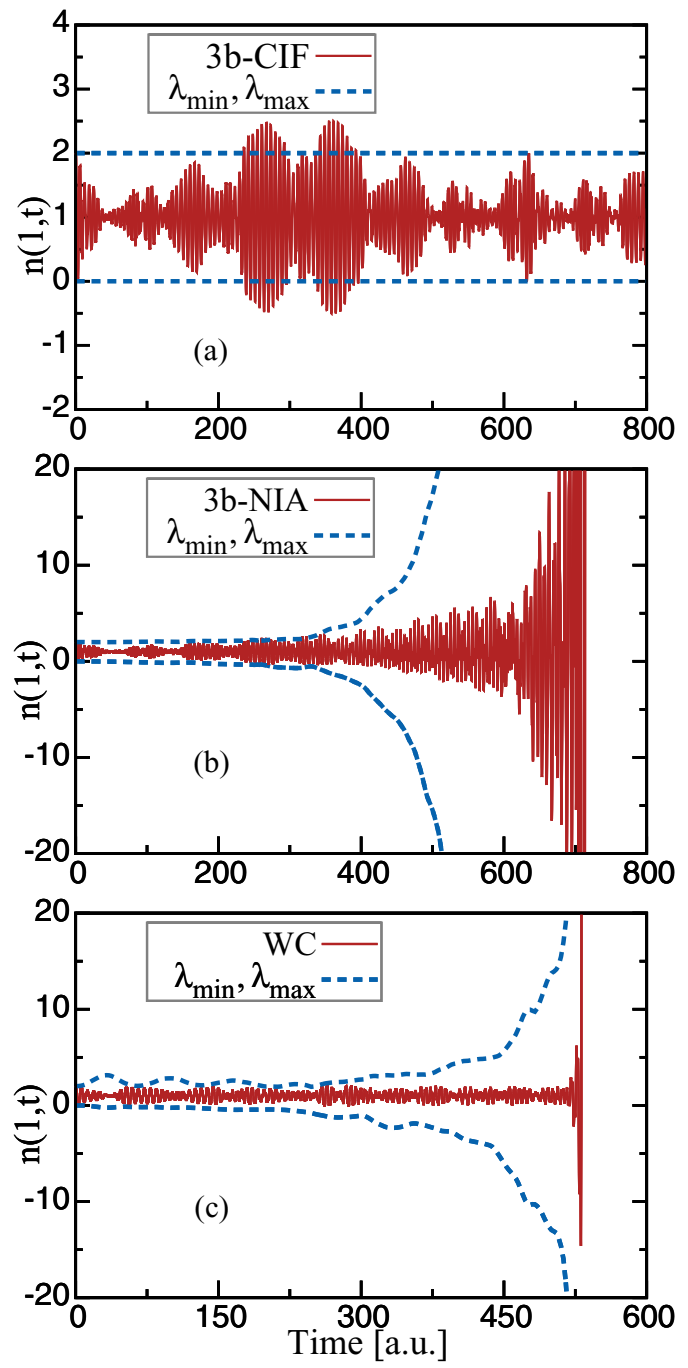

FIG. 2. (Color online) Time evolution of electronic density in the leftmost site of a four-site Hubbard model over a longer time scale for the (a) 3b-CIF, (b) 3b-NIA, and (c) WC approximations. Blue lines show the highest and lowest geminal occupation number in time. Here, $m, \hbar$ are set to unity and Hubbard parameters are $U=0.1$ and $t=1$. The four electrons filled the two leftmost sites initially.

the initial $\gamma$ and $\Gamma$ are positive semidefinite) but, since Eqs. (9) and (10) are not compatible and the relation between $\Gamma$ and $\gamma$ is ill defined, the positive semidefiniteness will not necessarily pass to $\gamma$ (see case A below).

It is not easy to impose positive semidefiniteness on $\Gamma^{(3)}$, and even if it has such a feature, since its trace relation with $\gamma$ and $\Gamma$ is broken, this does not lead to the positive semidefiniteness of $\gamma$ and $\Gamma$. In the following, we use different test approximations to analyze the role of compatibility and positive semidefiniteness in these unphysical results.

\section{A. Retaining positive semidefiniteness but not compatibility}

For the simplest case, 3b-CIF, the compatibility between the second and first equations of the hierarchy is obviously lost. At the same time, it is easy to show that the time evolution of $\Gamma$ is unitary in the sense that the solution to the equation of motion is of the form

$$
\Gamma\left(X_{2}, X_{2}^{\prime}, t\right)=\sum_{j} \lambda_{j} \Phi_{j}\left(X_{2}, t\right) \Phi_{j}^{*}\left(X_{2}^{\prime}, t\right),
$$

where $\Phi_{j}$ satisfies the Schrödinger equation

$$
i \partial_{t} \Phi_{j}\left(X_{2}, t\right)=\hat{H}_{12} \Phi_{j}\left(X_{2}, t\right) .
$$

Inserting Eq. (31) into Eq. (6) leads to time-independent geminal occupation numbers, and provided the initial $\Gamma$ is positive semidefinite, it preserves this feature at all times [Fig. 2(a)]. Note that this argument holds independent of possible time dependence in the two-particle Hamiltonian $\hat{H}_{12}$.

In addition, substituting Eq. (31) in the right-hand side of Eq. (9) yields a simple linear equation for the time propagation of $\gamma\left(\mathbf{x}_{1}, \mathbf{x}_{1}^{\prime}, t\right)$, which does not diverge. Nevertheless, because the partial trace relation [Eq. (5)] between $\gamma$ and $\Gamma$ is no longer valid, natural orbital occupation numbers may not necessarily lie between 0 and $1,{ }^{23}$ and as a consequence the density can gain negative values [Fig. 2(a)]. With this example we come to the conclusion that positive semidefiniteness of $\Gamma$ is not a sufficient condition for keeping $\gamma$ positive semidefinite.

\section{B. Retaining compatibility and positive semidefiniteness}

It is very insightful to look for an approximation that retains both of these properties and see how well it can perform in the calculation. The simplest possible approach is to replace the three-body collision integral term, $S$ in Eq. (10), with another term under the condition that the two equations become compatible. This condition, of course, does not determine a unique term, but to study its effect we consider the following simple expression:

$$
S\left(X_{2}, X_{2}^{\prime}, t\right)=(N-2)\left[U\left(X_{2}\right)-U\left(X_{2}^{\prime}\right)\right] \Gamma\left(X_{2}, X_{2}^{\prime}, t\right) .
$$

Thus, the second equation of the hierarchy becomes

$$
\begin{aligned}
& \left(i \partial_{t}-\hat{H}_{12}+\hat{H}_{1^{\prime} 2^{\prime}}\right) \Gamma\left(X_{2}, X_{2}^{\prime}, t\right) \\
& \quad=(N-2)\left[U\left(X_{2}\right)-U\left(X_{2}^{\prime}\right)\right] \Gamma\left(X_{2}, X_{2}^{\prime}, t\right) .
\end{aligned}
$$

When, in above equation, we set $\mathbf{x}_{2}=\mathbf{x}_{2}^{\prime}$ and integrate over $\mathbf{x}_{2}$, we obtain the first equation of the BBGKY hierarchy, Eq. (9).

This approximation makes Eq. (10) linear. Moreover, it retains the compatibility between the two equations, meaning the two approaches for solving the BBGKY hierarchy, discussed in Sec. II B, are equivalent. We call this the compatible approximation. The effect of this approximation is equivalent to magnifying the interparticle interaction by a factor of $(N-1)$ in the 3b-CIF approximation. Hence, we can modify interparticle interaction accordingly in Eq. (31) and use the same argument to prove that this approximation also keeps $\Gamma$ positive semidefinite. Now, as a result of compatibility, $\gamma=\gamma_{\Gamma}$, and this $\gamma$ immediately inherits the positive semidefiniteness of $\Gamma$; in this fashion, compatibility and positive semidefiniteness are both incorporated. The results for this approximation are depicted in the Fig. 3 for a limited time scale. As expected, we do not observe any violation in the electronic density even after a long propagation.

Despite the well-behaved result, it should be mentioned that although the natural orbital occupation numbers cannot acquire negative values, they may still exceed 1 (the density at one point may develop beyond two particles). For example, 


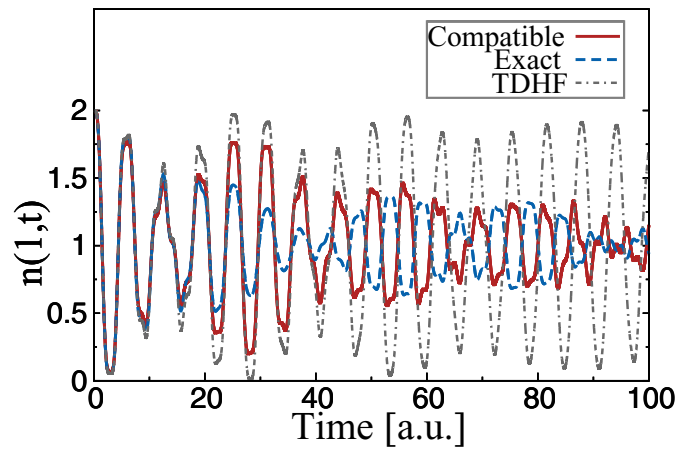

FIG. 3. (Color online) Number of electrons in the leftmost site of a four-site Hubbard model with the compatible approximation. The exact and TDHF results are also given for comparison. Here, $m$ and $\hbar$ are set to unity, and the Hubbard parameters are $U=0.1$ and $t=1$. The four electrons filled the two leftmost sites initially.

we observed such a violation for natural orbital occupation numbers in a tiny time region only when the initial state was far from equilibrium, i.e., four electrons occupying the two leftmost sites, and $U$ was very large $(U=10)$.

\section{Retaining compatibility but not positive semidefiniteness}

In order to find out the role of positive semidefiniteness and its importance, in the next step, we build an approximation that fulfills the compatibility but not positive semidefiniteness. To accomplish this goal, we add a term, $Z$, with a partial trace summing up to zero, to the previous compatible approximation, Eq. (33). Substituting this term in Eq. (10), we have

$$
\begin{aligned}
& \left(i \partial_{t}-\hat{H}_{12}+\hat{H}_{1^{\prime} 2^{\prime}}\right) \Gamma\left(X_{2}, X_{2}^{\prime}, t\right) \\
& \quad=(N-2)\left[U\left(X_{2}\right)-U\left(X_{2}^{\prime}\right)\right] \Gamma\left(X_{2}, X_{2}^{\prime}, t\right)+Z\left(X_{2}, X_{2}^{\prime}, t\right),
\end{aligned}
$$

where

$$
\int d \mathbf{x}_{2} Z\left(\mathbf{x}_{1}, \mathbf{x}_{2}, \mathbf{x}_{1}^{\prime}, \mathbf{x}_{2}, t\right)=0
$$

in most cases, $Z$ is a nonlinear functional of density matrices. In this way, both levels of the hierarchy remain compatible, but the additional term does not necessarily keep $\Gamma$ positive semidefinite. This term is not unique; nevertheless, there is a systematic approach to derive a possible form of it for different approximations (see Appendix $\mathrm{C}$ for a detailed derivation).

Nonetheless, although these approximations retain the compatibility, they do not necessarily keep positive semidefiniteness of RDMs, and they eventually lead to divergence if we propagate them long enough in time. These results are the evidence that compatibility is not a sufficient condition to keep the equations bounded.

\section{Using only the second equation}

As we discussed in Sec. IIB in detail, there are two approaches to solve the BBGKY hierarchy in the second level. So far in all discussed approximations, we have propagated both Eqs. (9) and (10) together; now, we turn to the second approach and solve only Eq. (10) for the above approximations.

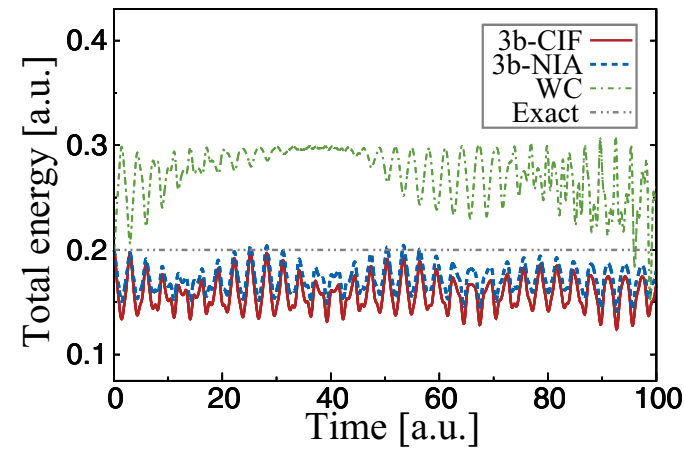

FIG. 4. (Color online) Fluctuations of the total energy of the foursite Hubbard model for different approximations using only Eq. (10). The exact result is also given for comparison. Here, $m$ and $\hbar$ are set to unity, and the Hubbard parameters are $U=0.1$ and $t=1$. Four electrons filled the two leftmost sites initially.

However, as we showed earlier, the total energy will not be necessarily conserved in this method unless the approximation makes the two equations compatible. Figure 4 shows that the energy fluctuations in time are relatively large for all the approximations, which puts a question mark over the quality of this approach in general.

Nonetheless, in this way, the compatibility between equations is not an issue any longer, which helps us to study the behavior of only the second equation. Our calculations show that in the case of the 3b-CIF approximation, despite a well-behaved electronic density, the natural orbital occupation numbers have violated mildly the upper bound of fermionic inequality. However, the divergence problem in the 3b-NIA or WC approximations has surfaced again, and in fact, the divergence time has not been improved in any of them, with these observations, we can confidently claim that the incompatibility is not the only cause of the diverging behavior of the equations, and the role of positive semidefiniteness should also be taken into account.

In these four sections, we have provided many test approximations fulfilling different constrains to show that neither compatibility between equations nor positive semidefiniteness of the approximations by itself can keep the propagation of the RDMs inside the fermionic boundaries. In fact, although the nonlinearity introduced by most of the approximations to Eq. (10) might be the cause of the divergence, the violation of the fermionic inequality might exist even in the case of linear approximations, as we saw in the $3 \mathrm{~b}$-CIF approximation. Therefore, it indeed takes both of these approximation constraints to tame such coupled equations.

\section{SUMMARY AND CONCLUSIONS}

In this work, we pointed out the main challenges we face in order to decouple the hierarchy of equations for the time evolution of density matrices. In particular, we studied several approximations for the three-body RDM in terms of the one- and two-body RDMs. First, we showed that once an approximation for the three-body RDM is made, the equation of motion for the two-body RDM no longer implies the validity 
of the first equation of the hierarchy. Therefore, in order to obey energy conservation it is necessary to solve the equations of motion for the one-body and two-body density matrices simultaneously.

Next, we studied numerical solutions for the fermionic Hubbard model for several of the decoupling schemes and compared them to the exact results obtained from solving the time-dependent Schrödinger equation. We found that in many decoupling schemes the local electron density attains unphysical negative values in time and natural orbital occupation numbers in general do not remain between 0 and 1 , as is required for fermionic systems. Furthermore, in most of existing approximations the local electron density diverges, although total particle number and energy are perfectly conserved. We investigated whether the divergence problem is solved by forcing the two lowest equations of the hierarchy to be compatible and found this not to be the case.

We conclude that a possible way to make progress in the application of the BBGKY hierarchy is to make sure that positive semidefiniteness of the density matrices and the fermionic constraints on the occupation numbers is built into the equations. For example, in the case of the time-dependent Hartree-Fock theory, there exists always a wave function corresponding to the first equation of the hierarchy, and therefore the natural orbital occupation numbers can never be unphysical. This suggests a further study of the BBGKY equations in relation to (approximate) wave functions. Further progress can be made in linear response theory since in the linear response regime the nonlinearities are, by definition, removed, and the BBGKY approach can be investigated further for the study of optical spectra. Work along these lines is in progress.

\section{ACKNOWLEDGMENTS}

The authors wish to thank E. K. U. Gross for useful discussions. We are also grateful to Ilya Tokatly and Ari Harju for their comments on the manuscript. We acknowledge financial support from the European Research Council Advanced Grant DYNamo (ERC-2010-AdG, Proposal No. 267374), Spanish Grants No. FIS2011-65702-C02-01 and No. PIB2010US00652, ACI-Promociona (Grant No. ACI2009-1036), Grupos Consolidados UPV/EHU del Gobierno Vasco (Grant No. IT-319-07), Consolider nanoTHERM (Grant No. CSD201000044), Fondo Social Europeo (FSE) and the Spanish Research Council (CSIC) through their "Junta para la Ampliación de Estudios" fellowship (JAE-Predoc-2008), and European Commission projects CRONOS (280879-2 CRONOS CPFP7) and THEMA (FP7-NMP-2008-SMALL-2, 228539). We are also grateful to the support of the Academy of Finland through its "Center of Excellence" Grant (2006-2011).

\section{APPENDIX A: ENERGY CONSERVATION DERIVATION}

Now we discuss in full detail the energy conservation in the BBGKY hierarchy. According to Eqs. (11) and (12), the change in the total energy of the system, $E(t)=E_{1}(t)+E_{2}(t)$, is given by

$$
\frac{d E}{d t}=\frac{d E_{1}}{d t}+\frac{d E_{2}}{d t},
$$

where

$$
\begin{aligned}
\frac{d E_{1}}{d t}= & \left.\int d \mathbf{x}^{\prime} \partial_{t} v\left(\mathbf{x}^{\prime}, t\right) \gamma\left(\mathbf{x}, \mathbf{x}^{\prime}, t\right)\right|_{\mathbf{x}=\mathbf{x}^{\prime}} \\
& +\left.\int d \mathbf{x}^{\prime} h\left(\mathbf{x}^{\prime}, t\right) \partial_{t} \gamma\left(\mathbf{x}, \mathbf{x}^{\prime}, t\right)\right|_{\mathbf{x}=\mathbf{x}^{\prime}}
\end{aligned}
$$

and

$$
\frac{d E_{2}}{d t}=\frac{1}{2} \int d \mathbf{x}_{1} d \mathbf{x}_{2} U\left(X_{2}\right) \partial_{t} \Gamma\left(X_{2}, X_{2}, t\right) .
$$

To proceed, we replace the time derivative of $\Gamma$ from Eq. (10); however, for $\gamma$ in Eq. (A2), we can use either Eq. (9) or Eq. (18). In our derivation, we use Eq. (18), and the case of Eq. (9) is obtained as the special case by taking $\bar{\Gamma}=\Gamma$ at the end of the derivation. Let us start by evaluating (A3). The equation of motion (10) yields

$$
\begin{aligned}
\frac{d E_{2}}{d t} & \\
= & -\frac{1}{4 i} \int d \mathbf{x}_{1} d \mathbf{x}_{2} U\left(X_{2}\right)\left(\nabla_{1}^{2}+\nabla_{2}^{2}-\nabla_{1^{\prime}}^{2}-\nabla_{2^{\prime}}^{2}\right) \\
& \times\left.\Gamma\left(X_{2}, X_{2}^{\prime}, t\right)\right|_{1,2=1^{\prime}, 2^{\prime}} \\
= & -\left.\frac{1}{2 i} \int d \mathbf{x}_{1} d \mathbf{x}_{2} U\left(\mathbf{x}_{1} \mathbf{x}_{2}\right)\left(\nabla_{1}^{2}-\nabla_{1^{\prime}}^{2}\right) \Gamma\left(\mathbf{x}_{1} \mathbf{x}_{2}, \mathbf{x}_{1}^{\prime} \mathbf{x}_{2}, t\right)\right|_{1=1^{\prime}} \\
= & \frac{1}{2 i} \int d \mathbf{x}_{1} \nabla_{1} U\left(\mathbf{x}_{1} \mathbf{x}_{2}\right) \cdot\left[\left.\left(\nabla_{1}-\nabla_{1^{\prime}}\right) \Gamma\left(\mathbf{x}_{1} \mathbf{x}_{2}, \mathbf{x}_{1}^{\prime} \mathbf{x}_{2}, t\right)\right|_{1=1^{\prime}}\right]
\end{aligned}
$$

where we used the symmetry of $\Gamma$ and performed a partial integration. Now, we consider the change in the one-body energy $E_{1}(t)$, primarily by evaluating the second term in Eq. (A2). It reads

$$
\begin{aligned}
\int & \left.d \mathbf{x} h\left(\mathbf{x}^{\prime}, t\right) \partial_{t} \gamma_{\Gamma}\left(\mathbf{x}, \mathbf{x}^{\prime}, t\right)\right|_{\mathbf{x}=\mathbf{x}^{\prime}} \\
= & \int d \mathbf{x}\left[v(\mathbf{x}, t) \partial_{t} n_{\Gamma}(\mathbf{x}, t)+\left.\frac{1}{2} \partial_{t} \nabla \cdot \nabla^{\prime} \gamma_{\Gamma}\left(\mathbf{x}, \mathbf{x}^{\prime}, t\right)\right|_{\mathbf{x}=\mathbf{x}^{\prime}}\right],
\end{aligned}
$$

where in the second term on the right-hand side, we used

$$
\begin{aligned}
& \int d \mathbf{x} d \mathbf{x}^{\prime} \delta\left(\mathbf{x}-\mathbf{x}^{\prime}\right) \nabla^{\prime 2} \gamma_{\Gamma}\left(\mathbf{x}, \mathbf{x}^{\prime}, t\right) \\
& =-\int d \mathbf{x} d \mathbf{x}^{\prime} \delta\left(\mathbf{x}-\mathbf{x}^{\prime}\right) \nabla \cdot \nabla^{\prime} \gamma_{\Gamma}\left(\mathbf{x}, \mathbf{x}^{\prime}, t\right) .
\end{aligned}
$$

On the other hand, from the equation of motion (18) it follows that

$$
\begin{aligned}
&\left.i \partial_{t} \nabla_{1} \cdot \nabla_{1^{\prime}} \gamma_{\Gamma}\left(\mathbf{x}_{1}, \mathbf{x}_{1^{\prime}}, t\right)\right|_{1=1^{\prime}} \\
&= {\left.\left[\nabla_{1} v\left(\mathbf{x}_{1}, t\right) \cdot \nabla_{1^{\prime}}-\nabla_{1^{\prime}} v\left(\mathbf{x}_{1}^{\prime}, t\right) \cdot \nabla_{1}\right] \gamma_{\Gamma}\left(\mathbf{x}_{1}, \mathbf{x}_{1^{\prime}}, t\right)\right|_{1=1^{\prime}} } \\
&-\frac{1}{2} \nabla_{1} \cdot\left[\left.\left(\nabla-\nabla^{\prime}\right)\left(\nabla_{1} \cdot \nabla_{1^{\prime}}\right) \gamma_{\Gamma}\left(\mathbf{x}, \mathbf{x}^{\prime}, t\right)\right|_{1^{\prime}=1^{\prime}}\right] \\
&+\left(\nabla_{1} \cdot \nabla_{1^{\prime}}\right) \int d \mathbf{x}_{2}\left[U\left(\mathbf{x}_{1} \mathbf{x}_{2}\right)-U\left(\mathbf{x}_{1}^{\prime} \mathbf{x}_{2}\right)\right] \\
& \times\left.\bar{\Gamma}\left(\mathbf{x}_{1} \mathbf{x}_{2}, \mathbf{x}_{1}^{\prime} \mathbf{x}_{2}, t\right)\right|_{1=1^{\prime}} .
\end{aligned}
$$


The second term on the right-hand side of this expression is a total derivative and vanishes upon integration. Putting Eq. (A7) back into Eq. (A5) and the resulting expression in Eq. (A2), we get

$$
\begin{aligned}
\frac{d E_{1}}{d t}= & \int d \mathbf{x}\left\{\partial_{t} v(\mathbf{x}, t) n_{\Gamma}(\mathbf{x}, t)\right. \\
& \left.+v(\mathbf{x}, t)\left[\partial_{t} n_{\Gamma}(\mathbf{x}, t)+\nabla \cdot \mathbf{j}_{\Gamma}(\mathbf{x}, t)\right]\right\} \\
& +\frac{1}{2 i} \int d \mathbf{x}_{1} d \mathbf{x}_{2}\left[\nabla_{1} U\left(\mathbf{x}_{1} \mathbf{x}_{2}\right) \cdot \nabla_{1^{\prime}}-\nabla_{1^{\prime}} U\left(\mathbf{x}_{1}^{\prime} \mathbf{x}_{2}\right) \cdot \nabla_{1}\right] \\
& \times\left.\bar{\Gamma}\left(\mathbf{x}_{1} \mathbf{x}_{2}, \mathbf{x}_{1}^{\prime} \mathbf{x}_{2}, t\right)\right|_{1=1^{\prime}} .
\end{aligned}
$$

The second term under the first integral vanishes since the continuity equation holds. Therefore, for the total energy we finally arrive at

$$
\begin{aligned}
\frac{d E}{d t}= & \int d \mathbf{x} \partial_{t} v(\mathbf{x}, t) n_{\Gamma}(\mathbf{x}, t)+\frac{1}{2 i} \int d \mathbf{x}_{1} d \mathbf{x}_{2} \nabla_{1} U\left(\mathbf{x}_{1} \mathbf{x}_{2}\right) \\
& \cdot\left\{\left.\left(\nabla_{1}-\nabla_{1^{\prime}}\right)\left[\Gamma\left(\mathbf{x}_{1} \mathbf{x}_{2}, \mathbf{x}_{1}^{\prime} \mathbf{x}_{2}, t\right)-\bar{\Gamma}\left(\mathbf{x}_{1} \mathbf{x}_{2}, \mathbf{x}_{1}^{\prime} \mathbf{x}_{2}, t\right)\right]\right|_{1=1^{\prime}}\right\}
\end{aligned}
$$

In the case that $\gamma$ evolves through Eq. (9), we must replace $\bar{\Gamma}$ with $\Gamma$, and hence the final result reads

$$
\frac{d E}{d t}=\int d \mathbf{x} \partial_{t} v(\mathbf{x}, t) n(\mathbf{x}, t),
$$

which is exactly the energy conservation law as discussed in the main text.

\section{APPENDIX B: COMPUTATIONAL DETAILS FOR THE HUBBARD MODEL}

The real-space description of Eqs. (9) and (10) makes them very impractical for computational purposes. Therefore, they must be transformed to the matrix form, using an appropriate basis set. In the case of the Hubbard model, the basis set is made of site orbitals for each spin; for example, for a four-site Hubbard system, the basis set contains eight orbitals.

Here we only concern ourselves with spin-compensated systems (i.e., $S_{z}=S=0$ ), in which we can use many symmetries to eliminate the spins and simplify the equations of motion. ${ }^{44}$ We define

$$
\gamma\left(\mathbf{r}_{1} \mathbf{r}_{1}^{\prime}, t\right)=\sum_{\sigma_{1}} \gamma\left(\mathbf{r}_{1} \sigma_{1}, \mathbf{r}_{1}^{\prime} \sigma_{1}, t\right)
$$

and

$$
\Gamma\left(\mathbf{r}_{1} \mathbf{r}_{2}, \mathbf{r}_{1}^{\prime} \mathbf{r}_{2}^{\prime}, t\right)=\sum_{\sigma_{1} \sigma_{2}} \Gamma\left(\mathbf{r}_{1} \sigma_{1} \mathbf{r}_{2} \sigma_{2}, \mathbf{r}_{1}^{\prime} \sigma_{1} \mathbf{r}_{2}^{\prime} \sigma_{2}, t\right),
$$

where $\sigma$ denotes the spin coordinate. Therefore, the matrix form of Eqs. (9) and (10) for an $M$-site Hubbard model in the site-orbital basis set reads

$$
\begin{aligned}
i \partial_{t} \gamma_{i j}= & t\left[\gamma_{i(j+1)}\left(1-\delta_{j M}\right)+\gamma_{i(j-1)}\left(1-\delta_{j 1}\right)\right. \\
& \left.-\gamma_{(i+1) j}\left(1-\delta_{i M}\right)-\gamma_{(i-1) j}\left(1-\delta_{i 1}\right)\right] \\
& +U\left(\Gamma_{i j j j}-\Gamma_{i i j i}\right)
\end{aligned}
$$

and

$$
\begin{aligned}
& i \partial_{t} \Gamma_{i j k l}=t\left[\Gamma_{i j(k+1) l}\left(1-\delta_{k M}\right)+\Gamma_{i j(k+1) l}\left(1-\delta_{k 1}\right)+\Gamma_{i j k(l+1)}\left(1-\delta_{l M}\right)+\Gamma_{i j k(l-1)}\left(1-\delta_{l 1}\right)\right. \\
& \left.-\Gamma_{(i+1) j k l}\left(1-\delta_{i M}\right)-\Gamma_{(i-1) j k l}\left(1-\delta_{i 1}\right)-\Gamma_{i(j+1) k l}\left(1-\delta_{j M}\right)-\Gamma_{i(j-1) k l}\left(1-\delta_{j 1}\right)\right]
\end{aligned}
$$

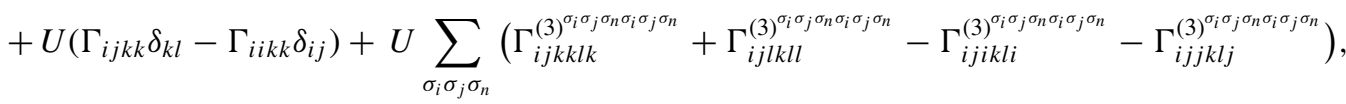

where the indices denote the site numbers. $\Gamma^{(3)}$ should be replaced by an approximation, and then the spins can be integrated out using the same symmetry argument. These are the actual equations that we solve in this work.

As mentioned in the main text, we choose a four-site Hubbard model where four electrons initially filled the two leftmost sites so that

$$
\gamma_{i, j}= \begin{cases}\gamma_{i, i}=2, & i=1,2, \\ \gamma_{i, j}=0, & \text { otherwise. }\end{cases}
$$

Now, since this initial state is a Slater determinant formed by two site orbitals, $\Gamma$ has the exact form of Eq. (23) and can be written as

$$
\Gamma_{i j k l}=\gamma_{i, k} \gamma_{j, l}-\frac{1}{2} \gamma_{i, l} \gamma_{j, k}
$$

For the initial state being the ground state of the HartreeFock approximation, we use Eq. (B6) with the same argument and construct $\Gamma$ from $\gamma$. Evidently, we cannot use the same argument for any initial state. In the case of starting from the exact ground state, we extract the exact $\gamma$ and $\Gamma$ and feed them into the equations.

\section{APPENDIX C: DERIVING THE ZERO-TRACED TERM}

Here, we derive the zero-traced expression $Z$, which we introduced in Sec. III C. This additional term can be written for different $\Gamma^{(3)}$ approximations as

$$
\begin{aligned}
Z\left(X_{2}, X_{2}^{\prime}, t\right)= & \int d \mathbf{x}_{3}\left[U\left(\mathbf{x}_{1} \mathbf{x}_{3}\right)+U\left(\mathbf{x}_{2} \mathbf{x}_{3}\right)\right. \\
& \left.-U\left(\mathbf{x}_{1}^{\prime} \mathbf{x}_{3}\right)-U\left(\mathbf{x}_{2}^{\prime} \mathbf{x}_{3}\right)\right] \Gamma_{\text {app }}^{(3)}\left(\mathbf{x}_{1} \mathbf{x}_{2} \mathbf{x}_{3}, \mathbf{x}_{1}^{\prime} \mathbf{x}_{2}^{\prime} \mathbf{x}_{3}, t\right) \\
& -F_{\text {app }}(\gamma, \Gamma),
\end{aligned}
$$

where the first term is the three-body collision integral with $\Gamma^{(3)}$ substituted by an approximation, and $F_{\text {app }}(\gamma, \Gamma)$ is defined for that approximation as

$$
\begin{aligned}
\operatorname{Tr}_{\mathbf{x}_{2}}\left[F_{\mathrm{app}}(\gamma, \Gamma)\right]= & \int d \mathbf{x}_{3} d \mathbf{x}_{2}\left[U\left(\mathbf{x}_{1} \mathbf{x}_{3}\right)-U\left(\mathbf{x}_{1}^{\prime} \mathbf{x}_{3}\right)\right] \\
& \times \Gamma_{\text {app }}^{(3)}\left(\mathbf{x}_{1} \mathbf{x}_{2} \mathbf{x}_{3}, \mathbf{x}_{1}^{\prime} \mathbf{x}_{2} \mathbf{x}_{3}, t\right) .
\end{aligned}
$$

Evidently, the partial trace of $Z$ will be zero. 
As an example, we give the explicit form of $F_{3 \mathrm{~b}-\mathrm{NIA}}(\gamma, \Gamma)$ as

$$
\begin{aligned}
F_{3 \mathrm{~b}-\mathrm{NIA}}(\gamma, \Gamma)= & {\left[U\left(\mathbf{x}_{1} \mathbf{x}_{2}\right)-U\left(\mathbf{x}_{1}^{\prime} \mathbf{x}_{2}^{\prime}\right)\right]\left\{N \left[\gamma\left(\mathbf{x}_{1}, \mathbf{x}_{1}^{\prime}\right) \gamma\left(\mathbf{x}_{2}, \mathbf{x}_{2}^{\prime}\right)\right.\right.} \\
& \left.-\gamma\left(\mathbf{x}_{1}, \mathbf{x}_{2}^{\prime}\right) \gamma\left(\mathbf{x}_{2}, \mathbf{x}_{1}^{\prime}\right)\right]+g\left(\mathbf{x}_{1}, \mathbf{x}_{2}^{\prime}\right) \gamma\left(\mathbf{x}_{2}, \mathbf{x}_{1}^{\prime}\right) \\
& -g\left(\mathbf{x}_{2}, \mathbf{x}_{2}^{\prime}\right) \gamma\left(\mathbf{x}_{1}, \mathbf{x}_{1}^{\prime}\right)+g\left(\mathbf{x}_{2}, \mathbf{x}_{1}^{\prime}\right) \gamma\left(\mathbf{x}_{1}, \mathbf{x}_{2}^{\prime}\right) \\
& \left.-g\left(\mathbf{x}_{1}, \mathbf{x}_{1}^{\prime}\right) \gamma\left(\mathbf{x}_{2}, \mathbf{x}_{2}^{\prime}\right)\right\},
\end{aligned}
$$

where

$$
g\left(\mathbf{x}_{1}^{\prime}, \mathbf{x}_{1}\right)=\int d \mathbf{x}_{2} \gamma\left(\mathbf{x}_{1}^{\prime}, \mathbf{x}_{2}\right) \gamma\left(\mathbf{x}_{2}, \mathbf{x}_{1}\right)
$$

In the same way, $F(\gamma, \Gamma)$ can be obtained for other approximations, but we do not present them here. Therefore, the full expression to replace the three-body collision integral reads

$$
\begin{aligned}
S\left(X_{2}, X_{2}^{\prime}, t\right)= & (N-2)\left[U\left(X_{2}\right)-U\left(X_{2}^{\prime}\right)\right] \Gamma\left(X_{2}, X_{2}^{\prime}, t\right) \\
& +\int d \mathbf{x}_{3}\left[U\left(\mathbf{x}_{1} \mathbf{x}_{3}\right)+U\left(\mathbf{x}_{2} \mathbf{x}_{3}\right)-U\left(\mathbf{x}_{1}^{\prime} \mathbf{x}_{3}\right)\right. \\
& \left.-U\left(\mathbf{x}_{2}^{\prime} \mathbf{x}_{3}\right)\right] \Gamma_{\text {app }}^{(3)}\left(\mathbf{x}_{1} \mathbf{x}_{2} \mathbf{x}_{3}, \mathbf{x}_{1}^{\prime} \mathbf{x}_{2}^{\prime} \mathbf{x}_{3}, t\right)-F_{\text {app }}(\gamma, \Gamma),
\end{aligned}
$$

where the first term comes from Eq. (33). *ali.akbari@csic.es

${ }^{\dagger}$ The first two authors have contributed equally to this work.

${ }^{1} \mathrm{~J}$. Frenkel, Wave Mechanics, Advanced General Theory (Clarendon, Oxford, 1934).

${ }^{2}$ P. A. M. Dirac, Proc. Cambridge Philos. Soc. 26, 376 (1930).

${ }^{3}$ D. Thouless, Quantum Mechanics of Many-Body Systems (Academic, New York, 1961).

${ }^{4}$ L. Kadanoff and G. Baym, Quantum Statistical Mechanics: Green's Function Methods in Equilibrium and Nonequilibrium Problems (W.A. Benjamin, New York, 1962).

${ }^{5}$ P. Myöhänen, A. Stan, G. Stefanucci, and R. van Leeuwen, Phys. Rev. B 80, 115107 (2009).

${ }^{6}$ L. V. Keldysh, Zh. Eksp. Teor. Fiz. 47, 1515 (1964) [Sov. Phys. JETP 20, 1018 (1965)].

${ }^{7}$ A. Stan, N. E. Dahlen, and R. van Leeuwen, J. Chem. Phys. 130, 224101 (2009).

${ }^{8}$ K. S. Thygesen and A. Rubio, Phys. Rev. B 77, 115333 (2008).

${ }^{9}$ E. Runge and E. K. U. Gross, Phys. Rev. Lett. 52, 997 (1984).

${ }^{10} \mathrm{C}$. Ullrich, Time-Dependent Density-Functional Theory: Concepts and Applications (Oxford University Press, New York, 2012).

${ }^{11}$ M. Born and H. S. Green, Proc. R. Soc. London, Ser. A 191, 168 (1947).

${ }^{12}$ N. N. Bogoliubov, The Dynamical Theory in Statistical Physics (Hindustan, Delhi, 1965).

${ }^{13}$ J. Kirkwood, J. Chem. Phys. 14, 180 (1946).

${ }^{14}$ J. Yvon, Nucl. Phys. 4, 1 (1957).

${ }^{15}$ A. M. K. Müller, Phys. Lett. A 105, 446 (1984).

${ }^{16}$ M. Buijse and E. Baerends, Mol. Phys. 100, 401 (2002).

${ }^{17}$ O. Gritsenko, K. Pernal, and E. J. Baerends, J. Chem. Phys. 122, 204102 (2005).

${ }^{18}$ S. Sharma, J. K. Dewhurst, N. N. Lathiotakis, and E. K. U. Gross, Phys. Rev. B 78, 201103 (2008).

${ }^{19}$ E. Tölö and A. Harju, Phys. Rev. B 81, 075321 (2010).

${ }^{20}$ H. Appel and E. K. U. Gross, Europhys. Lett. 92, 23001 (2010).
${ }^{21}$ K. J. Schmitt, P. G. Reinhard, and C. Toepffer, Z. Phys. A 336, 123 (1990).

${ }^{22}$ T. Gherega, R. Krieg, P. G. Reinhard, and C. Toepffer, Nucl. Phys. A 560, 166 (1993).

${ }^{23}$ A. J. Coleman, Rev. Mod. Phys. 35, 668 (1963).

${ }^{24}$ M. Bonitz, Quantum Kinetic Theory (B. G. Teubner Stuttgart, Leipzig, 1998).

${ }^{25}$ K. Pernal, O. Gritsenko, and E. J. Baerends, Phys. Rev. A 75, 012506 (2007)

${ }^{26}$ S. J. Wang and W. Cassing, Ann. Phys. NY 159, 328 (1985).

${ }^{27}$ W. Cassing and S. J. Wang, Z. Phys. A 328, 423 (1987).

${ }^{28}$ W. Cassing, K. Niita, and S. J. Wang, Z. Phys. A 331, 439 (1988).

${ }^{29}$ W. Cassing and A. Pfitzner, Z. Phys. A 337, 175 (1990).

${ }^{30}$ W. Cassing and A. Pfitzner, Z. Phys. A 342, 161 (1992).

${ }^{31}$ D. A. Mazziotti, Phys. Rev. A 57, 4219 (1998).

${ }^{32}$ F. Colmenero, C. Pérez del Valle, and C. Valdemoro, Phys. Rev. A 47, 971 (1993).

${ }^{33}$ H. Nakatsuji and K. Yasuda, Phys. Rev. Lett. 76, 1039 (1996).

${ }^{34}$ D. A. Mazziotti, Phys. Rev. A 60, 3618 (1999).

${ }^{35}$ D. A. Mazziotti, Chem. Phys. Lett. 289, 419 (1998).

${ }^{36}$ M. J. Hashemi, A. Akbari, R. van Leeuwen, A. Rubio, and R. M. Nieminen (unpublished).

${ }^{37}$ For the code, contact the first author.

${ }^{38}$ L. Shampine, Numerical Solution of Ordinary Differential Equations (Chapman and Hall, London, 1994).

${ }^{39}$ D. A. Mazziotti, Phys. Rev. Lett. 97, 143002 (2006).

${ }^{40}$ K. J. Schmitt, J. Stat. Phys. 46, 283 (1987).

${ }^{41}$ R. Krieg, K. J. Schmitt, and C. Toepffer, J. Stat. Phys. 57, 267 (1989).

${ }^{42}$ R. Goldman and E. A. Frieman, J. Math. Phys. 8, 1410 (1967).

${ }^{43}$ J. R. Dorfman and E. G. D. Cohen, J. Math. Phys. 8, 282 (1967).

${ }^{44}$ R. McWeeny and Y. Mizuno, Proc. R. Soc. London, Ser. A 259, 554 (1961). 